\title{
دراسة تحليلية لاهم المتغيرات المؤثرة على اسعار لحوم الدواجن بمحافظة الثرقية

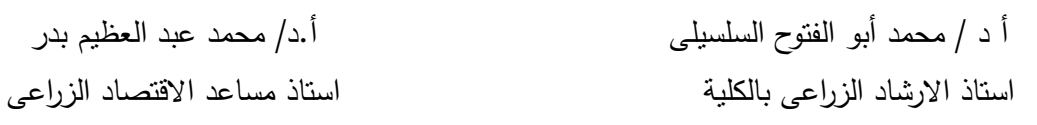

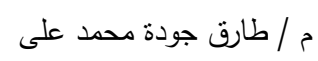

باحث بكلية الزراعة جامعة بنها باديا

Corresponding author: Tarekgouda916@gmail.com

مقدمة :

تعتبر صناعة الدواجن وخاصة دجاج اللحم أحد الأنشطة الإنتاجية الزراعية الهامة, فمن ناحية تعتبر أحد المصادر الرئيسية للاذل فى الإنتاج

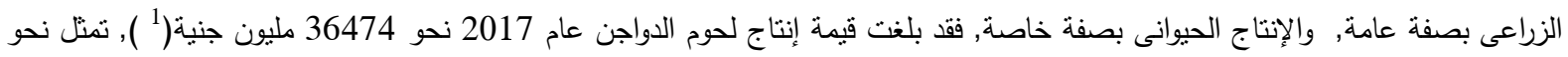
21.45\%من قيمة الإنتاج الحيوانى والبالغ نحو 169891مليون جنية , ومن ناحية آخرى تعتبر صناعة الدواجن أحد الركائز الرئيسية فى تحقيق

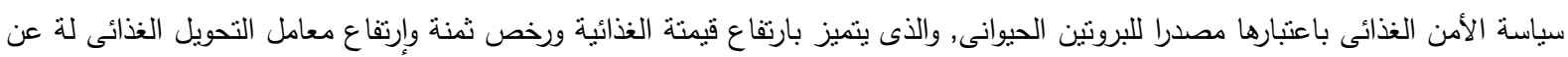

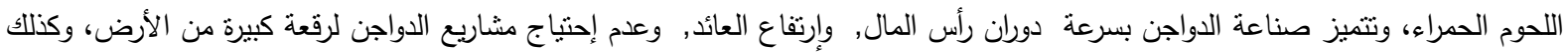

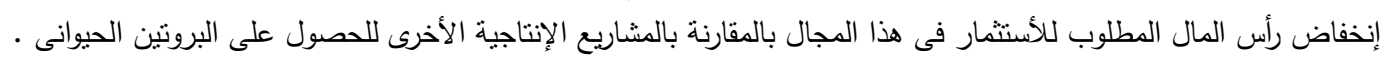

المشكلة البحثية:

تتحصر مشكلة البحث فى ارتفاع أسعار دجاج اللحم مما يستلزم تحليل أسعار دجاج اللحم لمعرفة اسباب الزيادة فى اسعار لحوم الدواجن

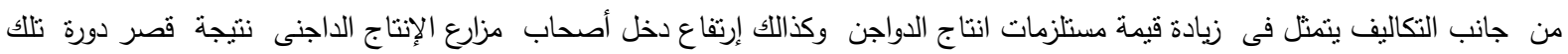

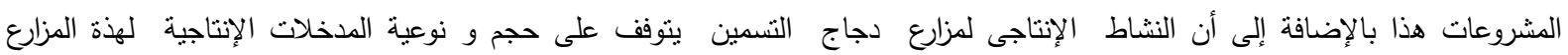

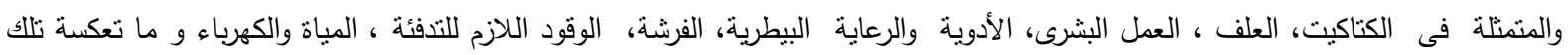

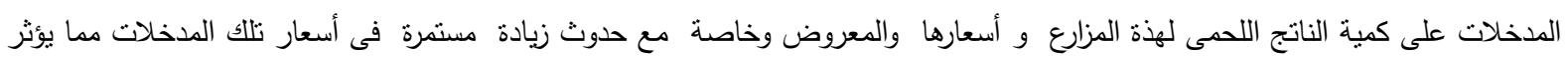

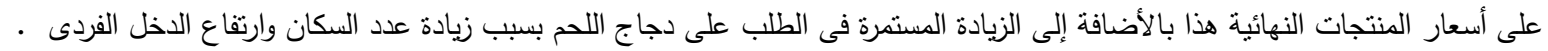

أهداف البحث:

لدراسة اهم المتغيرات المؤثرة على اسعار من خلال تحقيق الاهداف الفرعية الاتية: 1- التعرف على الاهمية النسبية لبنود التكاليف للانتا جفى مزارع تسمين البدارى وكذا تقدير دوال التكاليف الانتاجية لها. 2- التعرف على الحم انتاج الامثل فى السعات الانتاجية المدروسة. 3- تقدير مؤشرات الكفاءة الاقتصادية لمزارع التسمين المدروسة.

الطريقة البحثية و مصادر البيانات :

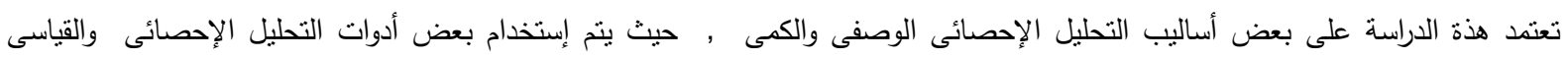

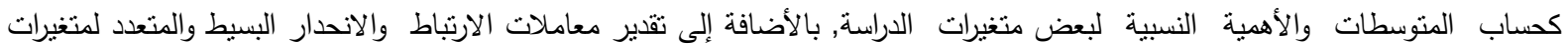

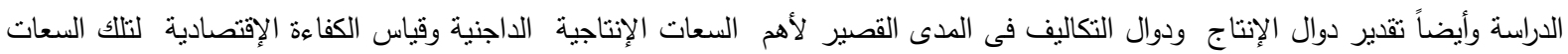

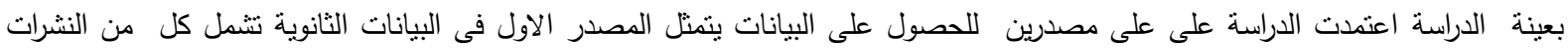

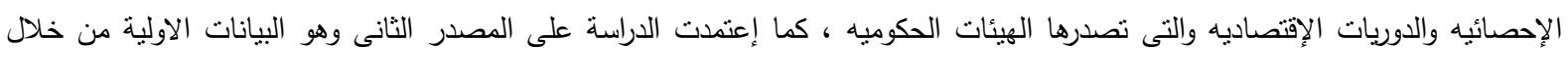
سحب عينة ميدانية من مزارع انتاج دجاج بدارى التسمين بمراكز محافظة الثرقية. 
فقد تم إجراء البحث عن طريق سحب عينة عشوائية طبقية من مزارع دجاج التسمين والتى تم تحديدها وفقا للأهمية النسبية للمراكز الإدارية بمحافظة الثرقية وحسب الأهمية النسبية لعدد مزارع دجاج التسمين فى نلك المراكز , ووقع الأختيار على اربعة مراكز من حيث الأهمية النسبية

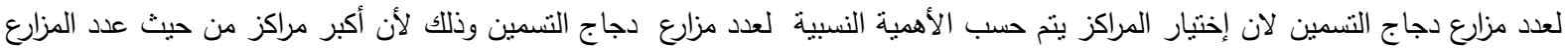

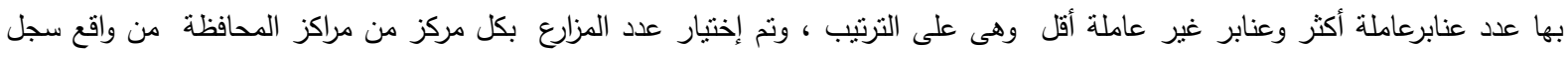

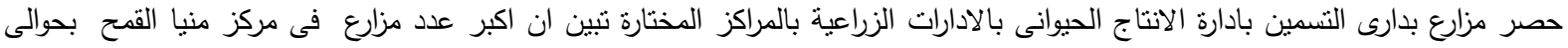

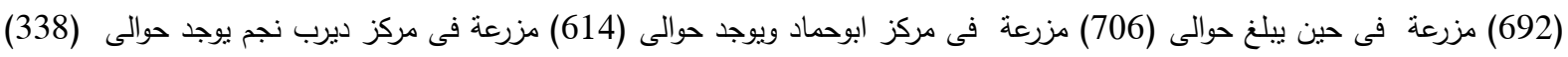

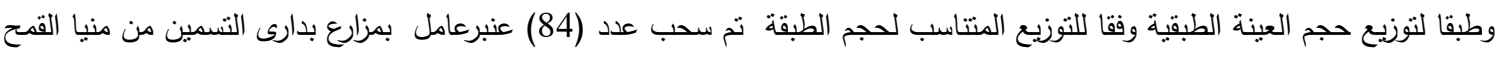
و عدد (78) عنبرعامل من مزارع بدارى التسمين من مركز ابوحماد و عدد (77) عنبر عامل من مزارع بدارى التسمين من مركز الحسينية و

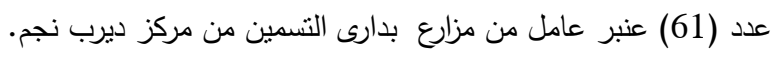

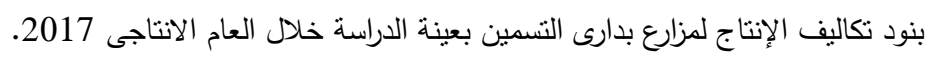

\section{النتائج ومناقتشتها:}

وبدراسة بنود تكاليف الانتاج لمزارع بدارى التسمين خلال منوسط عام انتاجى كامل بمتوسط خمس دورات تسمين خلال جدول ( 1 ) ) تبين من

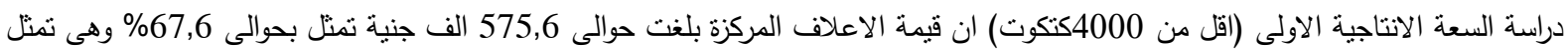

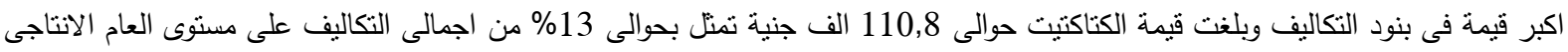

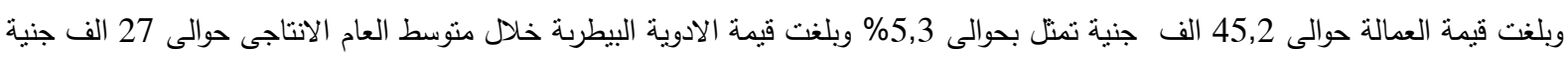

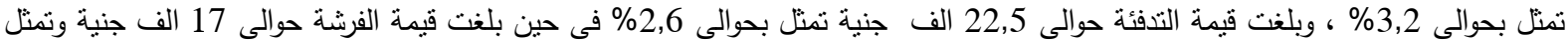

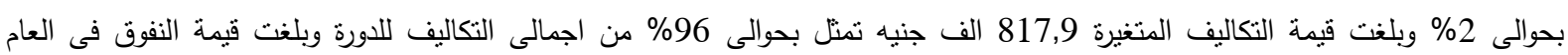

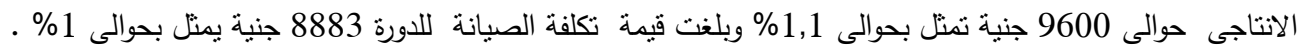
وتبين من دراسة السعة الانتاجية الثانية (4000كتكوت الى اقل من 6000كتكوت) ان قيمة الاعلاف المركزة بلغت حوالى 888,4 الف جنية الفية تمثل

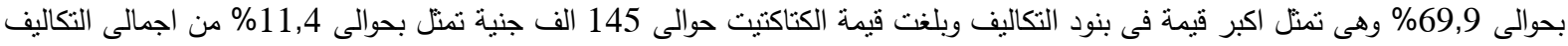
على مستوى الدورة الانتاجية وبلغت قيمة العمالة حوالى 66,5 الف جنية تمتل بحوالى 5,2\% وبلغت قيمة الادوية البيطربة حوالى 53,5 الف

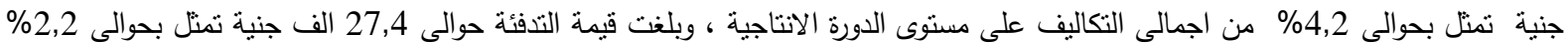

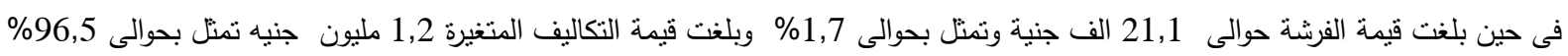
من اجمالى التكاليف للعام الانتاجى وبلغت قيمة النفوق للعام الانتاجى حوالى 2150 جنية تمثل بحوالى 1\% وبلى وبلغت قيمة نكلفة الصيانة للاورة

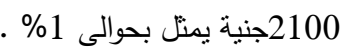

وتبين من دراسة السعة الانتاجية الثالثة ( 6000كتكوت الى اقل من 8000كتكوت) ان قيمة الاعلاف المركزة بلغت حوالى 1,2 مليون جنية تمنل

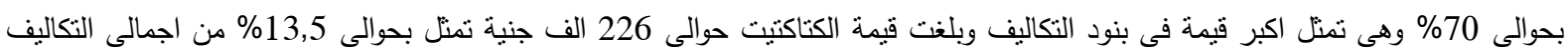

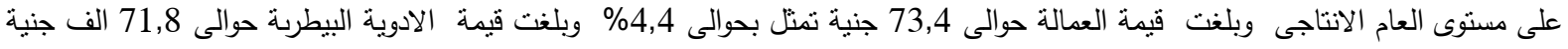

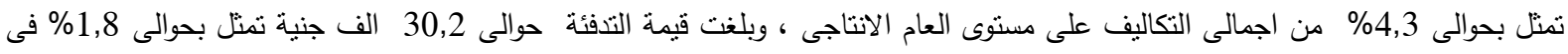

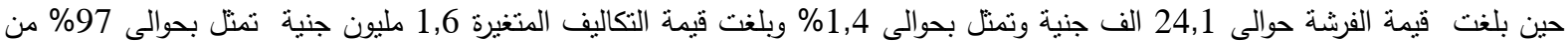
اجمالى التكاليف للاورة وبلغت قيمة النفوق للاورة حوالى 12,2 الف جنية تمثل بحوالى 0,7\% وبلغت قيمة تكلفة الصيانة للاورة 16,3جنية يمثل بحوالى 1\% . وتبين من دراسة السعة الانتاجية الرابعة (8000 كتكوت الى اقل من 1000كتكوت) ان قيمة الاعلاف المركزة بلغت حوالى 1,45 مليون جنية

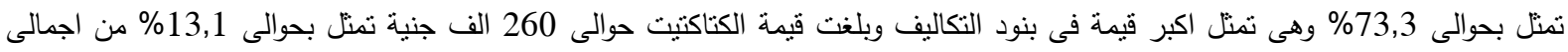

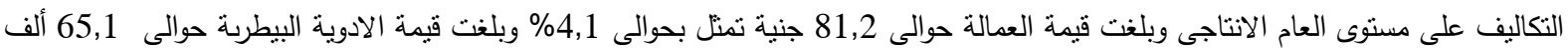

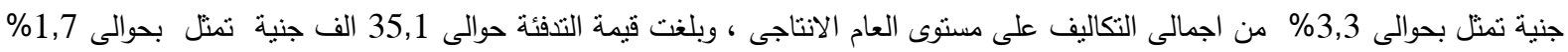

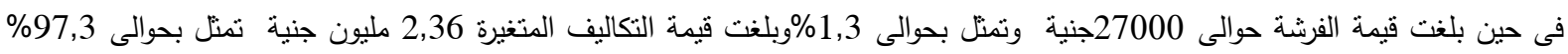

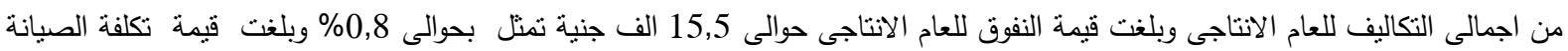
لللورة 17,6 الف جنية يمنل بحوالى 0,9\% وتبين من دراسة السعة الانتاجية الخامسة (10000كتكوت فاكثر) ان قيمة الاعلاف المركزة بلغت 
حوالى 292,8 الف جنية تمثل بحوالى 71,9\% وهى تمثل اكبر قيمة فى بنود التكاليف وبلغت قيمة الكتاكتيت حوالى 56000 جنية تمثل بحوالى 13,8\% من اجمالى التكاليف على مستوى الدورة الانتاجية وبلغت قيمة العمالة حوالى 15643 جنية تمثل بحوالى 3,8\% وبلغت قيمة الادوية البيطربة حوالى 14250جنية تمثل بحوالى 3,5\% من اجمالى التكاليف على مستوى الدورة الانتاجية ، وبلغت قيمة التدفئة حوالى 6742,5 جنية تمنل بحوالى 1,7 \% فى حين بلغت قيمة الفرشة حوالى 5000 جنية وتمنل بحوالى 1,2\%وبلغت قيمة التكاليف المتغيرة 396,65 كئل

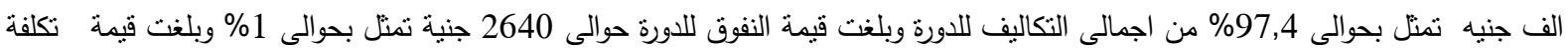

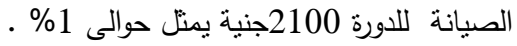

جدول رقم (1) :الأهمية النسبية لقيمة بنود نكاليف الإنتاج لمزارع تسمين البدارى بعينة الدراسة بمحافظة الثرقية لعام انتاجى كامل 2017.

\begin{tabular}{|c|c|c|c|c|c|c|c|c|c|c|}
\hline \multicolumn{2}{|c|}{ من الخامسة الانتاجية 10000 الى اقل 12000} & \multicolumn{2}{|c|}{ 8000الى اقل من الإجية الر ابعة 10000} & \multicolumn{2}{|c|}{ السعة الانتاجية الثالثة 8000 الثلة 8000} & \multicolumn{2}{|c|}{4000 اللى اقل الانتية الثانية 6000} & \multicolumn{2}{|c|}{ اقل من السعة الانتاجية الاولى كتكوت } & \multirow[t]{2}{*}{ السعة } \\
\hline$\%$ & المتوسط & $\%$ & المتوسط & $\%$ & المتوسط & $\%$ & المنوسط & $\%$ & المتوسط & \\
\hline 14.9 & $\begin{array}{c}356000 . \\
0\end{array}$ & 13.1 & $\begin{array}{c}260000 . \\
0\end{array}$ & 13.5 & $\begin{array}{c}226000 . \\
0\end{array}$ & 11.4 & $\begin{array}{c}145000 . \\
0\end{array}$ & 13.0 & $\begin{array}{c}110800 . \\
0\end{array}$ & قيمة الكتاكتيت \\
\hline 3.8 & 91858.0 & 4.1 & 81250.0 & 4.4 & 73425.0 & 5.2 & 66550.0 & 5.3 & 45250.0 & قيمة العمالة \\
\hline 72.1 & $\begin{array}{c}172680 \\
0.0\end{array}$ & 73.3 & $\begin{array}{c}145400 \\
0.0\end{array}$ & 70.0 & $\begin{array}{c}117260 \\
0.0\end{array}$ & 69.9 & $\begin{array}{c}888407 . \\
0\end{array}$ & 67.6 & $\begin{array}{c}575605 . \\
0\end{array}$ & قيمة الاعلاف المركزة \\
\hline 3.5 & 84500.0 & 3.3 & 65070.0 & 4.3 & 71780.0 & 4.2 & 53560.0 & 3.2 & 27010.0 & قيمة الادوية البيطرية \\
\hline 0.5 & 13000.0 & 0.6 & 12600.0 & 0.7 & 11500.0 & 0.9 & 11500.0 & 1.1 & 9200.0 & قيمة الاشر اف بيطرى \\
\hline 0.4 & 9100.0 & 0.4 & 8500.0 & 0.4 & 7220.0 & 0.5 & 6500.0 & 0.5 & 4340.0 & قيمة الكهرباء \\
\hline 0.2 & 4480.0 & 0.2 & 3200.0 & 0.1 & 1840.0 & 0.1 & 1600.0 & 0.2 & 1300.0 & قيمة المياه \\
\hline 0.2 & 5155.0 & 0.3 & 5155.0 & 1.8 & 30155.0 & 2.2 & 27374.0 & 2.6 & 22500.0 & قيمة التدفئة \\
\hline 1.3 & 31000.0 & 1.4 & 27100.0 & 1.4 & 24100.0 & 1.7 & 21100.0 & 2.0 & 17000.0 & قيمة الفرشة \\
\hline 0.3 & 8200.0 & 0.4 & 7700.0 & 0.4 & 6500.0 & 0.5 & 6100.0 & 0.6 & 4900.0 & قيمة الصيانة الدورية \\
\hline 97.3 & $\begin{array}{c}233009 \\
3.0\end{array}$ & 97.0 & $\begin{array}{c}192457 \\
5.0\end{array}$ & 97.0 & $\begin{array}{c}162512 \\
0.0\end{array}$ & 96.5 & $\begin{array}{c}122769 \\
1.0\end{array}$ & 96.0 & $\begin{array}{c}817905 . \\
0\end{array}$ & اجمالى التكاليف \\
\hline 0.8 & 19300.0 & 0.9 & 17600.0 & 1.0 & 16340.0 & 0.9 & 11600.0 & 1.0 & 8883.8 & قبمة تكلفة الصيانة \\
\hline 0.6 & 14900.0 & 0.6 & 11560.0 & 0.6 & 9500.0 & 0.7 & 8800.0 & 0.9 & 7900.0 & قيمة الايجار \\
\hline 0.6 & 15160.0 & 0.8 & 15700.0 & 0.7 & 11600.0 & 0.9 & 11800.0 & 0.9 & 7800.0 & قيمة العمالة الثابتة \\
\hline 0.6 & 15040.0 & 0.8 & 15500.0 & 0.7 & 12200.0 & 0.9 & 11900.0 & 1.1 & 9600.0 & قيمة النفوق \\
\hline 100.0 & $\begin{array}{c}239449 \\
3.0 \\
\end{array}$ & 100.0 & $\begin{array}{c}198493 \\
5.0 \\
\end{array}$ & 100.0 & $\begin{array}{c}167476 \\
0.0 \\
\end{array}$ & 100.0 & $\begin{array}{c}127179 \\
1.0 \\
\end{array}$ & 100.0 & $\begin{array}{c}852088 . \\
8 \\
\end{array}$ & اجمالى التكاليف \\
\hline
\end{tabular}

المصدر :_جمعت وحسبت باستخدام الحاسب الالى ، على بيانات أولية لدراسة ميدانية نم إجراؤها بمراكز عينة الدراسة ، خلال عام 2017

ثانيا - تقدير دوال التكاليف الإنتاجية في مزارع تسمين البدارى بمحافظة الشرقية : بدراسة الصور المختلفة لدوال التكاليف الإنتاجية في المدى القصير تبين أن الصورة التربيعية أفضل الصور من الناحيتين الاقتصادية والإحصائية , وهي أكثر الصور ملائمة لظروف الإنتاج الداجني . ومن خلالها أمكن حساب منوسط التكاليف الكلية والتكاليف الحدية , الحجم الأمنل للإنتاج في كل سعة وهو الحجم الذي تصل عنده متوسط التكاليف الكلية إلى أدنى مستوى لها وتتساوى مع التكاليف الحدية وكذللك يمكن حساب

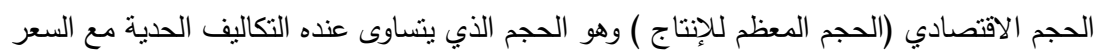
الثكل الرياضى للنموذج: يأخذ النموذج الصورة التربيعية التالية: $\mathrm{Y}_{\mathrm{X}}=\mathrm{b}_{0}+\mathrm{b}_{1} \mathrm{X}-\mathrm{b}_{2} \mathrm{X}^{2}$ 1- التقدير الإحصائي لدالة التكاليف لدجاج بدارى التسمين بالسعة الانتاجيةالأولى(اقل من 4000كتكوت) 
يتضح من جدول (2) نتائج تقدير دالة التكاليف فى الصورة التربيعية فى المدى القصير لانها اعطت افضل النتائج المكنة لتقدير دالة تكاليف

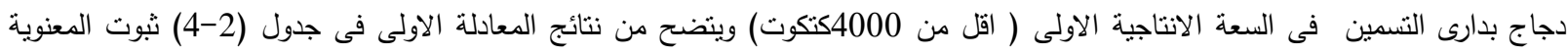

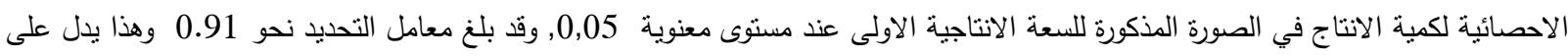
أن التغيرات فى كمية الإنتاج قد فسرت نحو 91\% من التغير في التكاليف الإنتاجية للسعة الأنتاجية الاولى (اقل من (F)

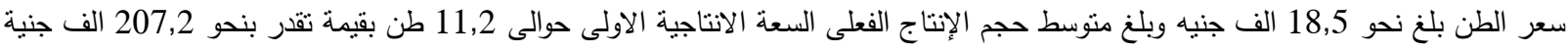

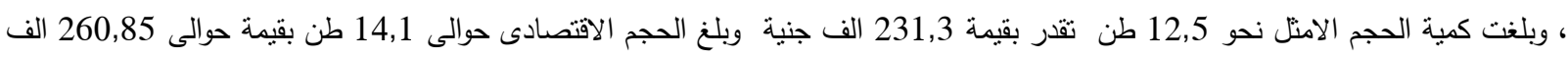
جنية ويرجع ذللك إلى انخفاض الجدارة الإنتاجية للمربيين في السعة الإنتاجية الاولى بالرغم من انخفاض الطاقة التشغيلية في تللك المزارع إلى حد

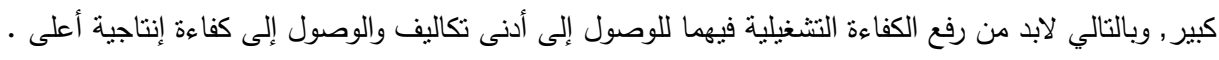

2- التقدير الإحصائي لدالة التكاليف لاجاج بدارى التسمين بالسعة الثانية (من

يتضح من جدول (2) نتائج تقدير دالة التكاليف فى الصورة التربيعية فى المدى القصبر لانها اعطت افضل النتائج الممكنة لتقدير دالة

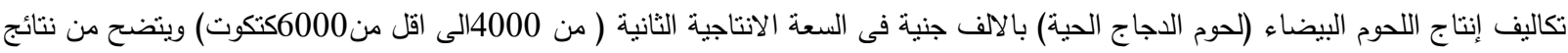
المعادلة الثانية فى جدول (2) ثبوت المعنوية الاحصائية لكمية الانتاج في الصورة المذكورة للسعة الانتاجية الثانية عند مستوى معنوية 0,05, وقد بلغ معامل التحديد نحو 0.89 وهذا يدل على أن التغيرات فى كمية الإنتاج قد فسرت نحو 89\% من التغير في التكاليف الإنتاجية للسعة الأنتاجية

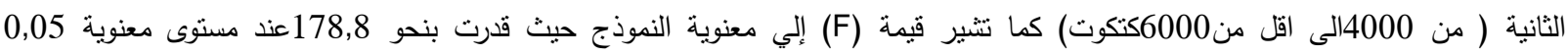
وللحصول على أكبرعائد اقتصادي من جدول (3) نجد أن متوسط سعر الطن بلغ نحو 18,4 الف جنيه وبلغ متوسط حجم الإنتاج الفعلى السعة الانتاجية الثانية حوالى 16,7 طن بقيمة تقدر بنحو 307,2 الف جنية ، وبلغت كمية الحجم الامثل نحو 18,9 طن تقدر بقيمة 347,8 الف جنية وبلغ الحجم الاقتصادى حوالى 20,2 طن بقيمة حوالى 371,7 الف جنية ويرجع ذلك إلى انخفاض الجدارة الإنتاجية للمربيين في السعة الإنتاجية الثانية بالرغم من انخفاض الطاقة التشغيلية في تلك المزارع إلى حد كبير , وبالتالي لابد من رفع الكفاءة النتغيلية فيهما للوصول إلى أدنى تكاليف والوصول إلى كفاءة إنتاجية أعلى من النفاضي

3- التقدير الإحصائي لدالة التكاليف لاجاج بدارى التسمين بالسعة الثالثة(6000كتكوت الى اقل من 8000 كتكوت) : يتضح من جدول (2) نتائج تقدير دالة التكاليف فى الصورة التربيعية فى المدى القصبر لانها اعطت افضل النتائج الممكنة لتقدير دالة تكاليف دجاج بدارى التسمين فى السعة الانتاجية الثالثة (6000كتكوت الى اقل من 8000 كتكوت) ويتضح من نتائج المعادلة الثالثة فى جدول (2) ثبوت المعنوية الاحصائية لكمية الانتاج في الصورة المذكورة للسعة الانتاجية الثالثة عند مستوى معنوية 0,05, وقد بلغ معامل التحديد نحو يدل على أن التغيرات فى كمية الإنتاج قد فسرت نحو 88\% من التغير في التكاليف الإنتاجية فى السعة الانتاجية الثالثة (6000كتكوت التى الثى اقل من 8000 كتكوت)، كما نتير قيمة (F) إلي معنوية النموذج حيث قدرت بنحو 223,6 عند مستوى معنوية 0,05 وللحصول على أكبر عائد اقتصادي من جدول (3) نجد أن منوسط سعر الطن بلغ نحو 18,2 الف جنيه وبلغ متوسط حجم الإنتاج الفعلى فى السعة الانتاجية الثالثة

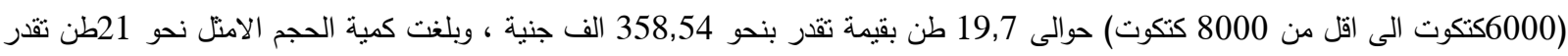
بقيمة 382,2 الف جنية وبلغ الحجم الاقتصادى حوالى 23,1 طن بقيمة حوالى 420,42 الف جنية ويرجع ذلك إلى انخفاض بلى بلى

4- التقدير الإحصائي لدالة التكاليف لاجاج بدارى التسمين بالسعة الرابعة(من 8000كتكوت الى اقل من 10000كتكوت) : يتضح من جدول (2) نتائج تقدير دالة التكاليف فى الصورة التربيعية فى المدى القصير لانها اعطت افضل النتائج المكنة لتقدير دالة تكاليف

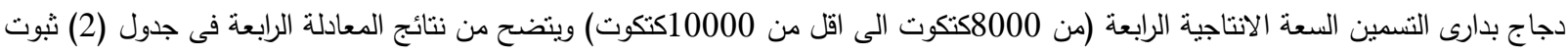

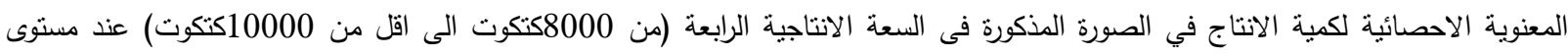
معنوية 0,05, وقد بلغ معامل التحديد نحو 0,91 وهذا يدل على أن التغيرات فى كمية الإنتاج قد فسرت نحو 91\% من الإنه التغير في التكاليف

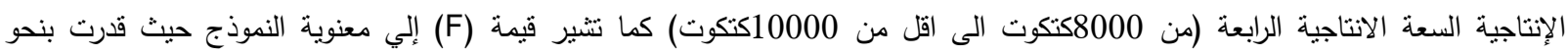
139,9 عند مستوى معنوية 0,05 وللحصول على أكبر عائد اقتصادي من جدول (3) نجد أن منوسط سعر الطن بلغ نحو 18,1 الف جنيه الفيه وبلغ

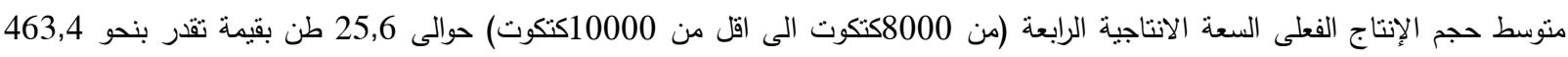

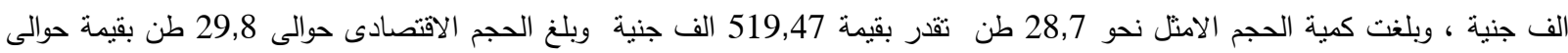

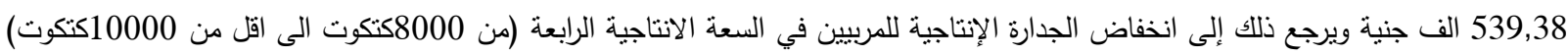
بالرغم من انخفاض الطاقة التتغيلية في تلك المزارع إلى حد كبير , وبالتالي لابد من رفع الكفاءة التشغيلية فيهما للوصول إلى أدنى تكاليف والوصول 


\begin{tabular}{|c|c|c|c|c|c|c|c|c|}
\hline $\mathrm{F}$ & $\mathrm{R}^{2}$ & $\mathrm{R}$ & $b^{2}$ & B & A & الدالية & المعادلة & السعة \\
\hline 121 & 0.91 & 0.58 & $\begin{array}{c}-28.7 \\
(-82.13)^{* *}\end{array}$ & 12345 & 35431 & خطية & 1 & 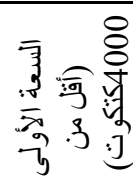 \\
\hline 178.8 & 089 & 0.68 & $(-92.13)^{* *}$ & $(62.13)^{* *}$ & $(32.93)^{* *}$ & قيمة ت & 2 & 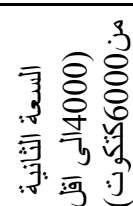 \\
\hline
\end{tabular}

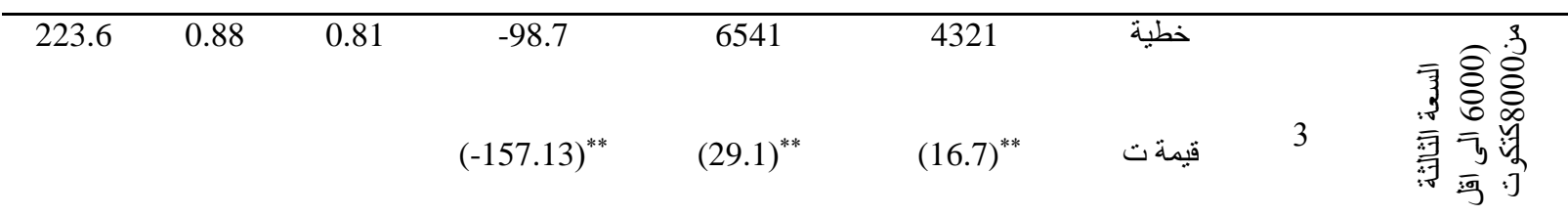

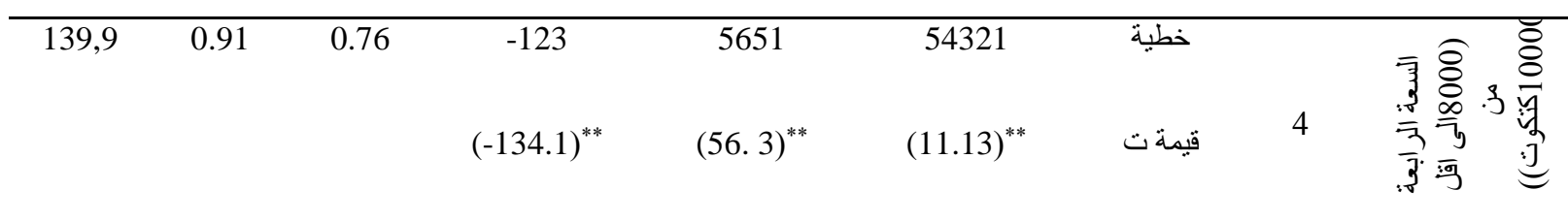

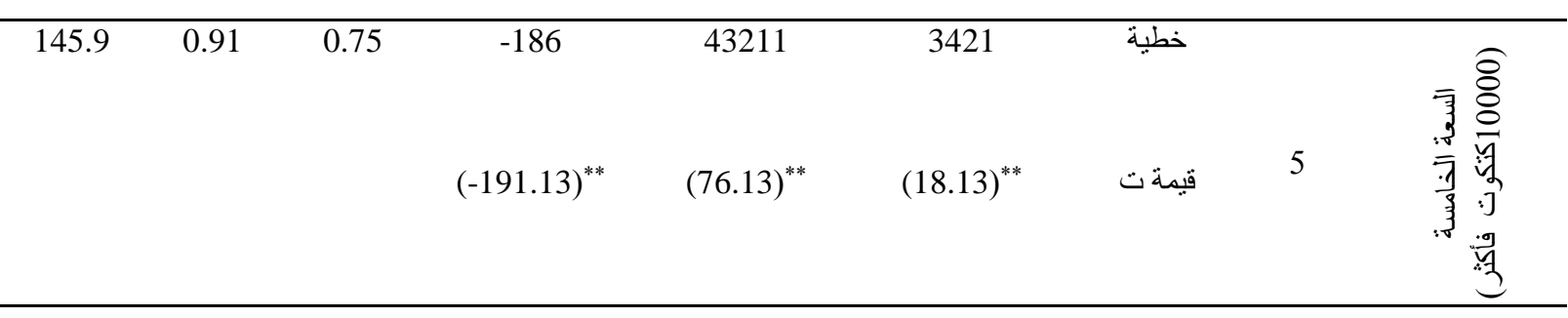

الصدر : جمعت وحسبت من بيانات استمارات الاستيان من مزارع بدارى التسمين بمحافظة الثرقيةعام 2017م

الجدارة الإنتاجية للمربين فى السعة الانتاجية الثالثة (6000كتكوت الى اقل من 8000 كنكوت) بالرغم من انخفاض الطاقة التتغيلية في تلك

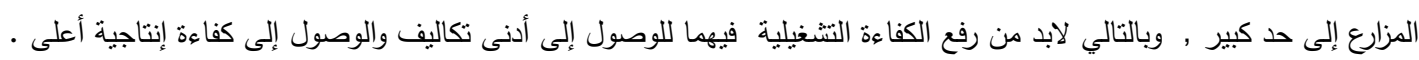

5- التقدير الإحصائي لدالة النكاليف لدجاج بدارى التسمين بالسعة الخامسة (10000كتكوت فأكثر) يتضح من جدول (2) نتائج تقدير دالة النكاليف فى الصورة التربيعية فى المدى القصير لانها اعطت افضل النتائج المكنة لتقدير دالة نكاليف

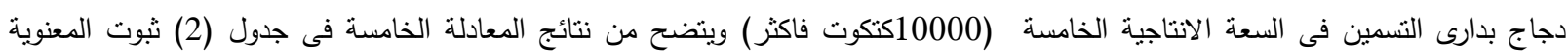
الاحصائية لكمية الانتاج في الصورة الدذكورة السعة الانتاجية الخامسة (10000كتكوت فاكثر) عند مستوى معنوية 
جدول رقم (3) : منتسط حجم الإنتاج والحجم الأمنل والحجم الاقتصادي في السعات الإنتاجية الخمسة لعينة الدراسة الميدانية للعام الإنتاجي 2017

\begin{tabular}{|c|c|c|c|c|c|c|c|}
\hline 'قتصادي & الحجم الا & لأمثل & الحجم & لإنتاج الفعلي & منتوسط حجم & 尔 & \\
\hline بالالف جنية ) & (الكمية بالطن) & (القيمة بالالف & (الكمية بالطن) & (القيمة بالالف & (الكمية بالطن) & 东 & $\therefore 8$ \\
\hline 260.85 & 14.1 & 231.25 & 12.5 & 207.2 & 11.2 & 18.5 & (أقل من السعة الأولى \\
\hline 371.68 & 20.2 & 347.76 & 18.9 & 307.28 & 16.7 & 18.4 & 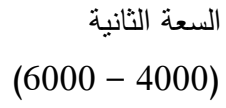 \\
\hline 420.42 & 23.1 & 382.2 & 21 & 358.54 & 19.7 & 18.2 & (6000 - 6000 السعة الثالثة \\
\hline 539.38 & 29.8 & 519.47 & 28.7 & 463.36 & 25.6 & 18.1 & السعة الرابعة \\
\hline 714.6 & 39.7 & 658.8 & 36.6 & 592.2 & 32.9 & 18 & (10000 السعة الخامسة \\
\hline 461.4 & 25.4 & 427.9 & 23.5 & 385.7 & 21.2 & 18.2 & متوسط العينة \\
\hline
\end{tabular}

0.05, وقد بلغ معامل التحديد نحو 0.91وهذا يدل على أن التغيرات فى كمية الإنتاج قد فسرت نحو 91\% من التغير في التكاليف الإنتاجية السعة الانتاجية الخامسة (10000كتكوت فاكثر) كما نشير قيمة (F) إلي معنوية النموذج حيث قدرت بنحو 139,9 عند مستوى معنوية 0,05 وللحصول على أكبر عائد اقتصادي من جدول (3) نجد أن منوسط سعر الطن بلغ نحو 18 الف جنيه وبلغ متوسط حجم الإنتاج الفعلى السعة الانتاجية الخامسة (10000كتكوت فاكثر) حوالى 32,2 طن بقيمة تقدر بنحو 592,2 الف جنية ، وبلغت كمية الحجم الامثل نحو 36,6 طن تقدر بقيمة 658,8 الف جنية وبلغ الحجم الاقتصادى حوالى 39,7 طن بقيمة حوالى 714,6 الف جنية ويرجع ذلك إلى انخفاض الجدارة الإنتاجية للمربيين في السعة الانتاجية الخامسة (10000كتكوت فاكثر) بالرغم من انخفاض الطاقة التتغيلية في تلك المزارع إلى حد كبير , وبالتالي لابد من

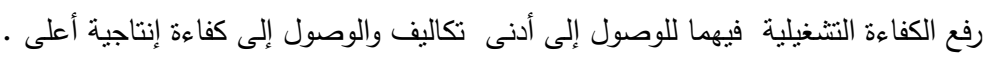

\section{تقدير مؤثرات الكفاعة الاقتصادية لمزارع تسمين البدارى بعينة الدراسة:}

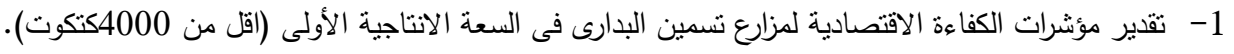

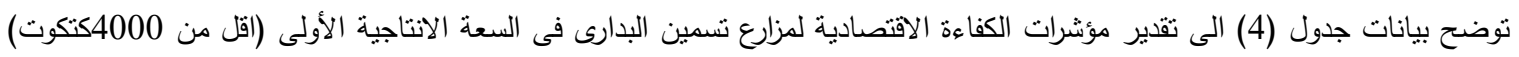

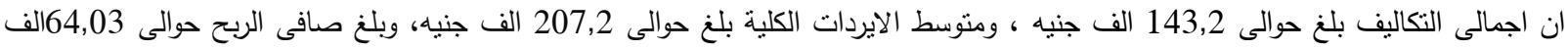
جنيه وبلغت اربحية الجنية المستتمر للمنتج حوالى 0,45 جنية وبلغت نسبة الايراد الكلى للتكاليف حوالى 1,44 ،كما بلغ معدل النمواليومى بالجرام حوالى 51,1 جرام وبلغ معدل الكفاءة الغذائية للعليقة حوالى 0,57 جرام فى حين بلغ منوسط تكلفة انتاج كجم اللحم حوالى 8,6 جنية / كجم 0

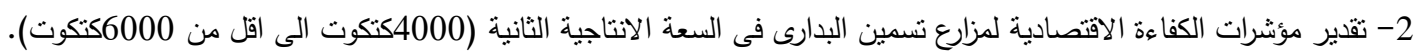
توضح بيانات جدول (4) الى تقدير مؤشرات الكفاءة الاقتصادية لمزارع تسمين البدارى فى السعة الانتاجية الثانية (4000كتكوت التى الى اقل من 6000كتكوت)ان اجمالى تكاليف تبلغ حوالى 209,8 الف جنيه ، ومنتوسط الايردات الكلية تبلغ حوالى 307,3 الف جنيه ،وبلغ صافى الربح حوالى 97,5الف جنيه وبلغت اربحية الجنية المستثمر للمنتج حوالى 0,46 جنية وبلغت نسبة الايراد الكلى للتكاليف حوالى 1,46، كما بلغ معدل النمو اليومى بالجم حوالى 51,6 جم وبلغ معدل التحويل الفنى للعليقة حوالى 0,56 جرام فى حين بلغ منوسط نكلفة انتاج كجم اللحم حوالى 8,9 جنية / كجم

3- تقدير مؤشرات الكفاءة الاقتصادية لمزارع تسمين البدارى فى السعة الانتاجية الثالثة (6000كتكوت الى اقل من 8000كتكوت). توضح بيانات جدول (4) الى تقدير مؤثرات الكفاءة الاقتصادية لمزارع تسمين البدارى فى السعة الانتاجية الثالثة (6000كتكوت الى اقل من اجمالى تكاليف تبلغ حوالى 277,7 الف جنيه ، ومتوسط الايردات الكلية تبلغ حوالى 358,54 الف جنيه ، وبلغ صافى الربح حوالى 80,8الف 
جنيه وبلغت اربحية الجنية المستثمر للمنتج حوالى 0,29 جنية وبلغت نسبة الايراد الكلى للتكاليف حوالى 1,29 ، كما كما بلغ معدل النمو اليومى بالجم

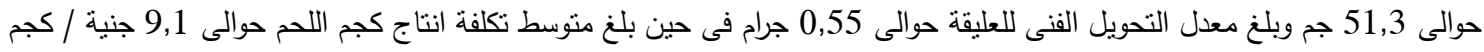

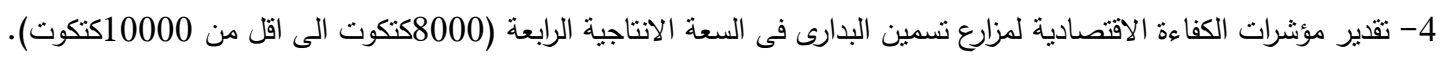

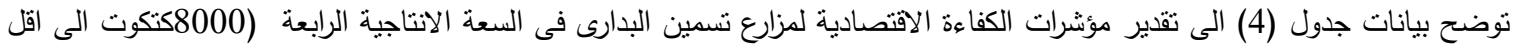

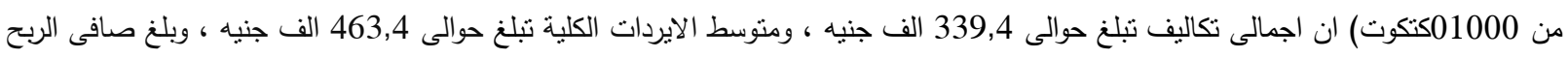

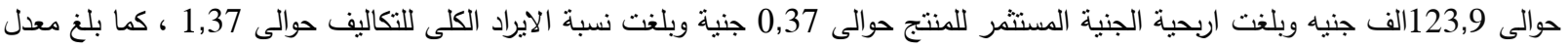

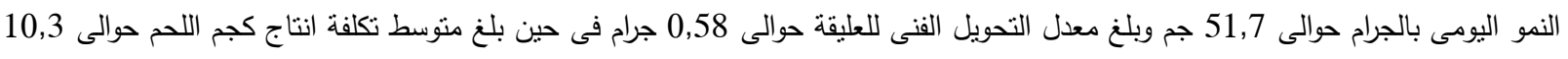

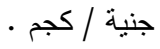

جدول رقم (4): بعض مؤثرات الكفاءة الاقتصادية لمزراع دجاج التسمين بالجنيه بعينة الدراسة الميدانية بمحافظة الثرقية للعام الزراعي 2017/2016

\begin{tabular}{|c|c|c|c|c|c|}
\hline$\frac{3}{3}$ & 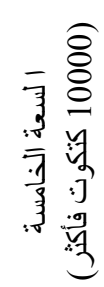 & 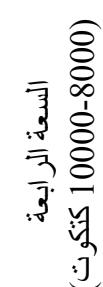 & 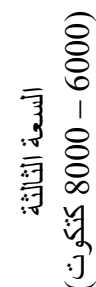 & 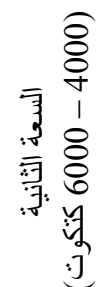 & 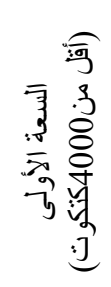 \\
\hline
\end{tabular}

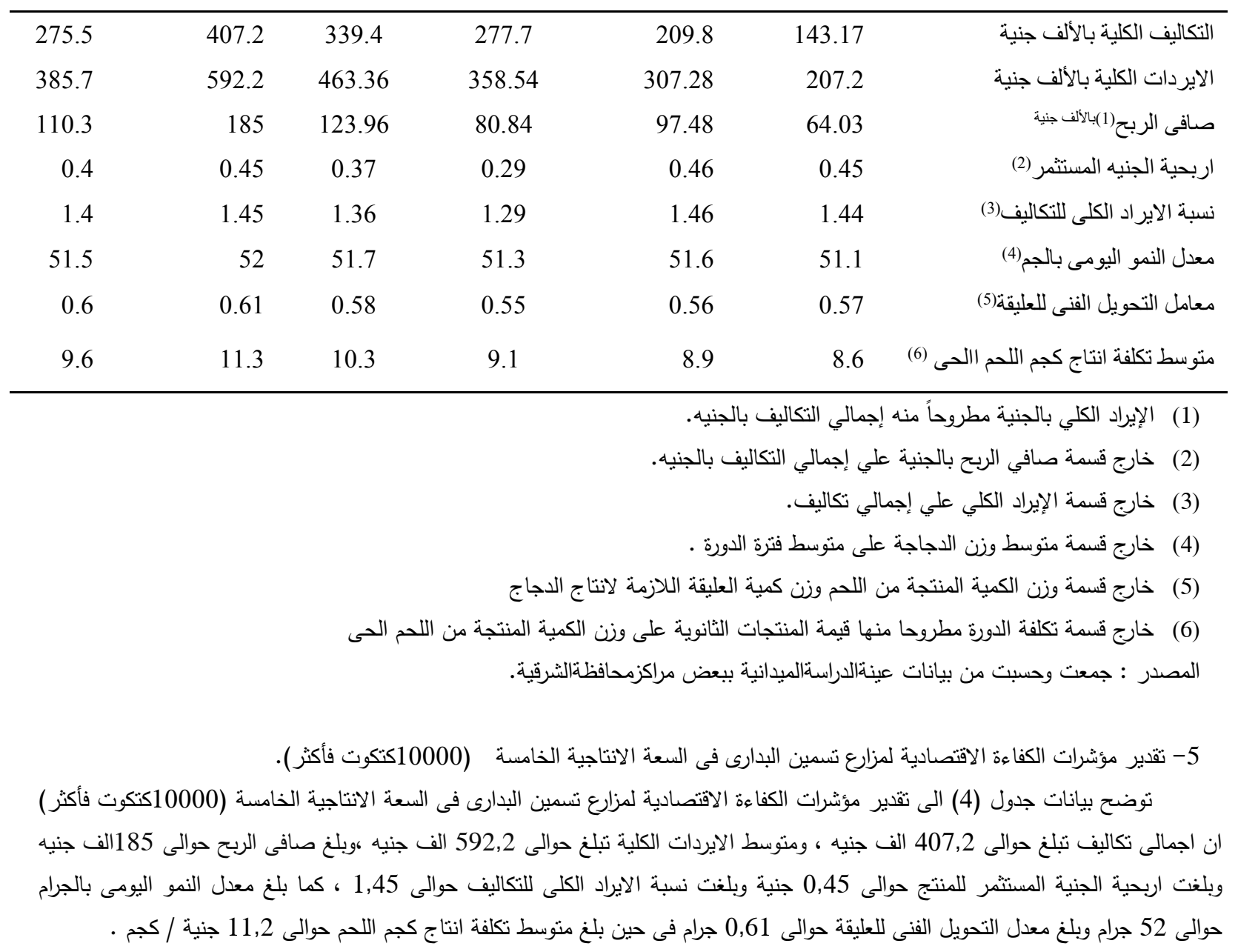


تعتبر صناعة الدواجن وخاصة دجاج اللحم أحد الأنشطة الإنتاجية الزراعية الهامة, فمن ناحية تعتبر أحد المصادر الرئيسية للاخل فى الإنتاج الزراعى بصفة عامة, والإنتاج الحيوانى بصفة خاصة وبدراسة بنود نكاليف الانتاج لمزارع بدارى التسمين خلال منوسط عام انتاجى كامل

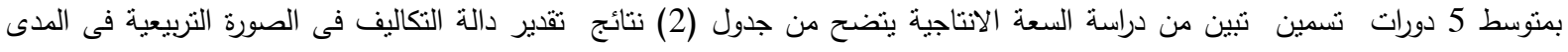

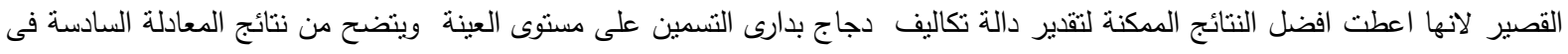
جدول (3) نجد أن متوسط سعر الطن بلغ نحو 18.2 الف جنيه وبلغ منوسط حجم الإنتاج الفعلى على مستوى العينة حوالى 21.2 طن بقيمة تقدر بنحو 385.7 الف جنية ، وبلغت كمية الحجم الامتل نحو 23.5 طن تقدر بقيمة 427.9 الف جنية وبلغ الحجم الاقتصادى حوالى 25.4 طن بقيمة حوالى 461.4 الف جنية ويرجع ذللك إلى انخفاض الجدارة الإنتاجية للمربين على مستوى العينة بالرغم من انخفاض الطاقة التشغيلية

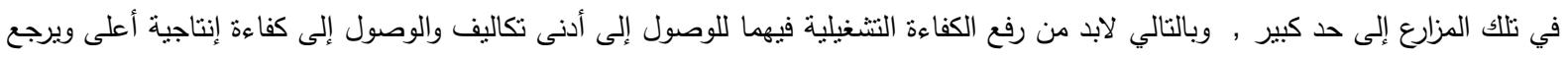

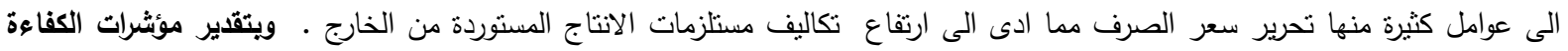

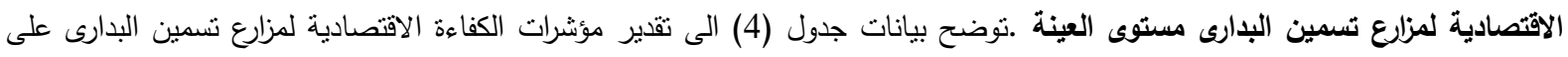

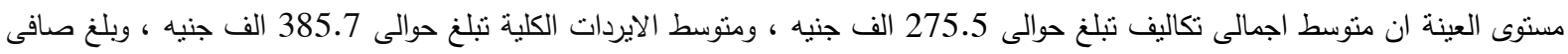
الربح حوالى 110.3الف جنيه وبلغت اربحية الجنية المستثمر للمنتج حوالى 0.4 جنية وبلغت نسبة الايراد الكلى التكاليف حوالى 1.4 ، كما بلغ

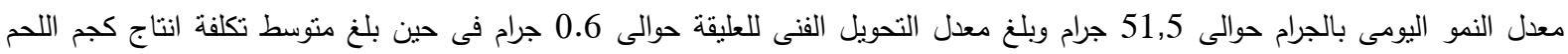

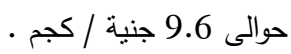
المراجع 1- مديرية الزراعة بالثرقية ، إدارة الإنتاج الحيواني ، بيانات غير منشورة . 2- عباس فتحى العوضى , دراسة تحليلية لأسعار الدواجن فى مصر ,المجلة المصرية للإقتصاد الزراعى ,المجلد الرابع عشر , العدد الثانى يونيو

3-محمد إمام عبد النبى (دكتور) , صناعة إنتاج بدارى اللحم وتنويقها فى مصر رمجلة المنصورة للعلوم الزاعية ,المجلد (24),والعدد (7)

$$
\begin{aligned}
& \text { 4- وزارة الزراعة واستصلاح الأراضي ، القطاع الاقتصادي ، نشرة الدخل الزراعي ، أعداد متفرقة }
\end{aligned}
$$

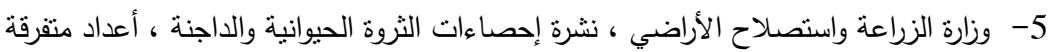

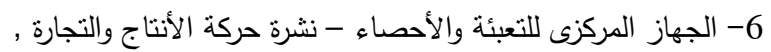

\section{An Analytical Study of the Most Important Variables Affecting Poultry Meat Prices in Sharkia Governorate}

The poultry industry, especially chicken meat, is an important agricultural production activity, on the one hand it is one of the main sources of income in agricultural production in general, Animal production in particular and the study of the production cost items of fattening farms during an average full production year with an average of 5 fattening cycles, the study of production capacity is shown from the table (2) the results of the estimate of the cost function in the quadratic picture in the short term because it gave the best possible results to estimate the function of the costs of chicken fattening at the sample level and is illustrated from the results of the sixth equation in table (3) we find that the average price of a ton was about 18.2 The average volume of actual production at the sample level was about 21.2 tons with an estimated value of 385.7 thousand pounds, and the optimal volume amounted to about 23.5 tons estimated at 427.9 thousand pounds and the economic volume was about 25.4 tons worth about 461.4 thousand pounds due to the decrease in the productive merit of the breeders at the sample level despite the decrease in the operational capacity of those farms to a large extent Therefore, the operational efficiency of them must be increased to reach the lowest costs and to reach higher productivity efficiency due to many factors, including the liberalization of the exchange rate, which led to higher costs of production supplies imported from abroad. By estimating the economic efficiency indicators of the farmers of fattening the badari sample level. 10.3 thousand pounds and the profitability of the pound invested for the product about 0.4 pounds and the ratio of total revenue cost about 1.4 , the daily growth rate in grams was about 51.5 grams and the technical conversion rate of the blackberry about 0.6 grams while the average cost of producing $\mathrm{kg}$ of meat was about 9.6 pounds $/ \mathrm{kg}$.

Keywords: chicken meat, Prices, Sharkia Governorate 